\title{
Sinonasal malignancies in Sweden 1960-2010; a nationwide study of the Swedish population*
}

\author{
Alexandra Elliot', Mattias Jangard' ${ }^{1}$, Linda Marklund', Niclas Håkansson², \\ Paul Dickman³, Lalle Hammarstedt-Nordenvall', Pär Stjärne' \\ Department of Oto-Rhino-Laryngology, Head and Neck Surgery, Karolinska Institutet, Karolinska University Hospital, Stockholm, \\ Sweden \\ ${ }^{2}$ Institute of Environmental Medicine Karolinska Institutet, Stockholm, Sweden \\ ${ }^{3}$ Institute of Medical Epidemiology and Biostatistics, Karolinska Institutet, Stockholm, Sweden
}

Rhinology 53: 75-80, 2015

DOI:10.4193/Rhino14.070

*Received for publication:

March 12, 2014

Accepted: September 4, 2014

\begin{abstract}
Introduction: Sinonasal malignancies (SNM) are rare and the prognosis is generally poor. Recently, a change in incidence for SNM has been reported. In this study, we investigated population-based trends for SNM in Sweden.
\end{abstract}

Methods: We identified 3,221 patients from the Swedish National Cancer Registry diagnosed with primary malignancies arising from the nasal cavity, paranasal sinuses, or both, during the period 1960 through 2010. Incidence, gender and age, anatomical sites and relative survival were investigated.

Results: The incidence for SNM decreased except for sinonasal malignant melanoma (SNMM) and adenoid cystic cancer (even though a very small group) during the study period. More than $50 \%$ of the malignancies involved the nasal cavity. The five-year relative survival was highest for adenoid cystic cancer followed by adenocarcinoma. SNMM and undifferentiated carcinoma had the poorest prognosis.

Conclusion: We found that the incidence for SNM has decreased during the study period 1960 through 2010, except for SNMM that has increased.

Key words: sinonasal, malignant, paranasal sinuses, incidence, survival

\section{Introduction}

Malignancies of the nasal cavity and the paranasal sinuses are rare, accounting for approximately $0.1 \%$ of all malignancies and $5 \%$ of all head and neck malignancies in Sweden ${ }^{(1)}$. The incidence of sinonasal malignancy (SNM), except sinonasal malignant melanoma (SNMM), has been reported to decrease since 1960 in Sweden. However, the reason for this is still unknown ${ }^{(2,3)}$. The decreasing incidence, except for squamous cell carcinoma (SCC) in women, has also been shown in a study in the Netherlands from $2012^{(4)}$. Another large study in the United States from 2011 found a relatively stable incidence the last 30 years ${ }^{(5)}$. Generally, these tumours have a poor prognosis as they often present at a locally advanced stage. Despite treatment, the 5-year survival rate for patients with SNMs is poor especially for undifferentiated tumours and SNMMs. Due to the scarcity of
SNMs, treatment in Sweden as well as internationally, has been based on local traditions. Therefore the effect of different treatment modalities on the survival is still uncertain ${ }^{(2,3,5,6)}$.

The purpose of the current study was to explore the incidence trends, the survival changes in different histological subtypes of SNMs, and gender related differences. We used the Swedish Cancer Registry (SCR), which due to the high registration rate harbours a unique opportunity to study incidence trends and prognosis of SNMs in Sweden over time. Our investigation included all sinonasal malignancies in Sweden registered from 1960 to 2010.

\section{Materials and methods}

Subjects

All patients in Sweden diagnosed with sinonasal malignancies 
from 1960-2010 were identified using the Swedish Cancer Registry (SCR). The SCR was established in 1958 by the National Board of Health and Welfare ${ }^{(1)}$. All clinicians and pathologists or cytologists diagnosing a primary case of cancer must by law file a report. The SCR covers approximately $96 \%$ of all cancer cases diagnosed in Sweden. Approximately $94 \%$ of the cases are morphologically verified. Since the reliability of the SCR is uncertain concerning the two first years, we used data from $1960^{(7,8)}$.

The SCR classifies reported cases using the ICD-7. Clinical diagnoses according to ICD-8 to ICD-10 have been transformed to ICD-7 in the register. We included all malignant tumours in the sino-nasal area, numbered 160.0, 160.2, 160.7-160.9 according to the ICD-7. Information extracted from the SCR included standard data such as, histological subtype, localization, age and date at diagnosis and gender. Information on clinical stage is only available in the SCR for patients diagnosed from 2004 and could not be used to study long-term temporal trends.

Using the emigration-, immigration- and date of death registers from Statistics Sweden, we were able to identify patients lost to follow-up and to analyse survival. For the survival analysis, we included patients diagnosed up to 2010 with follow-up to the end of 2012.

To calculate incidence rates, annual population- and gender distribution, data were also retrieved from Statistics Sweden (Statistical Yearbook of Sweden. Publication Services Statistics, Sweden, Annual Publications. Örebro).

The study was approved by the Regional Ethics Committee at the Karolinska Institute.

\section{Statistics}

The cancer cases were divided into 16 age groups according to age at diagnosis, each age span including five years, except for the oldest ( $\geq 85$ years). The calendar period was divided into fiveyear-periods beginning in 1960. Incidence rates were calculated by dividing the number of cases in each calendar period by the total average population in each age group in the respective calendar period. The incidence rates were calculated for the whole population as well as sex specific. For comparison, the rates are also adjusted to the Swedish Standard Population of year 2000 as well as the World Standard Population of 1966. Differences in the five-year periods incidence rates were analysed and their $\mathrm{p}$-values are given.

We estimated relative survival ratios as a measure of patient survival ${ }^{(9)}$. Relative survival provides a measure of excess mortality due to cancer without relying on cause of death information; it is defined as the observed all-cause survival in the patients divided by the expected survival of a comparable group from the general population. Expected survival was estimated using the Ederer II method from Swedish population life-tables stratified by age, sex, and calendar period. Poisson regression ${ }^{(10)}$ was used to model the effect of age at diagnosis, sex, and calendar year at diagnosis, on the excess mortality rate ratio. Relative survival was calculated as the ratio of the observed to the expected survival rate, in which the later was inferred from the survival of the entire Swedish population in the same age, sex and calendar year stratum. The age - standardized relative survival rate ${ }^{(11)}$ was also estimated using age distribution $(<55,55-69, \geq 70)$ at cancer diagnosis among all patients. To investigate independent effects of patient characteristics along with calendar period, we

Table 1. Characteristics of patients with sinonasal malignancies in Sweden 1960-2010.

\section{Patient characteristics}

All patients $(n=3221)$

\begin{tabular}{|cc|}
\hline Age, year & $n(\%)$ \\
\hline$<55$ & $581(18.0)$ \\
\hline $55-69$ & $1153(35.8)$ \\
\hline$\geq 70$ & $1487(46.2)$ \\
\hline Gender & \\
\hline Females & $1258(39.1)$ \\
\hline Male & $1963(60.9)$
\end{tabular}

\section{Histological subtype}

Squamous cell 1476 (45.8)

Adenocarcinoma

Melanoma

Adenoid cystic

Undifferentiated

494 (15.3)

Other

\section{Localization, anatomical site}

Nasal cavity $1608(49.9)$

Maxillary sinus $1012(31.4)$

Other sinus 340 (10.6)

Spread growth 94 (2.9)

Not specified 167 (5.2)

Age Years Range $0-100$ Mean 66.4 Median 68.0 
Table 2. Histology and topography of 3,221 patients with sinonasal malignancies in Sweden, 1960-2010.

\begin{tabular}{|c|c|c|c|c|c|c|c|c|c|c|c|}
\hline \multirow[b]{2}{*}{ Histology } & \multirow[b]{2}{*}{ All } & \multicolumn{2}{|c|}{ No. (\%) } & \multicolumn{2}{|c|}{ No. (\%) } & \multirow[b]{2}{*}{ Nasal cavity } & \multirow{2}{*}{$\begin{array}{l}\text { No. (\%) } \\
\text { Maxillary } \\
\text { sinus }\end{array}$} & \multirow[b]{2}{*}{ Other } & \multicolumn{3}{|c|}{ Mean age (Yrs) } \\
\hline & & Men & Women & Men & $\begin{array}{l}\text { Wo- } \\
\text { men }\end{array}$ & & & & All & Men & $\begin{array}{l}\text { Wo- } \\
\text { men }\end{array}$ \\
\hline Squamous cell carcinoma & 1476 & 930 (47.4\%) & $546(43.4 \%)$ & $63 \%$ & $37 \%$ & $713(48.3 \%)$ & $568(38.5 \%)$ & $195(13.2 \%)$ & 67.6 & 66.6 & 69.3 \\
\hline Adenocarcinoma & 393 & $300(15.3 \%)$ & $93(7.4 \%)$ & $76 \%$ & $24 \%$ & $178(45.3 \%)$ & $65(16.5 \%)$ & $150(38.2 \%)$ & 65.8 & 65.8 & 65.8 \\
\hline Melanoma & 306 & $137(7.0 \%)$ & 169 (13.4\%) & $45 \%$ & $55 \%$ & $250(81.7 \%)$ & $23(7.5 \%)$ & $33(10.8 \%)$ & 71.7 & 71.0 & 72.3 \\
\hline Adenoid cystic carcinoma & 129 & $60(3.1 \%)$ & $69(5.5 \%)$ & $47 \%$ & $53 \%$ & $50(38.8 \%)$ & $52(40.3 \%)$ & $27(20.9 \%)$ & 62.5 & 61.6 & 63.3 \\
\hline Undifferentiated & 494 & $298(15.2 \%)$ & $196(15.6 \%)$ & $60 \%$ & $40 \%$ & $187(37.9 \%)$ & $195(39.5 \%)$ & $112(22.7 \%)$ & 65.3 & 63.7 & 67.8 \\
\hline Other & 423 & $238(12.1 \%)$ & 185 (14.7\%) & $56 \%$ & $44 \%$ & $230(54.4 \%)$ & $109(25.8 \%)$ & $84(19.9 \%)$ & 61.5 & 58.5 & 65.4 \\
\hline Total & 3221 & $1,963(100 \%)$ & $1,258(100 \%)$ & $61 \%$ & $39 \%$ & $1,608(49.9 \%)$ & $1,012(31.4 \%)$ & 601 (18.7\%) & 66.4 & 65.2 & 68.3 \\
\hline
\end{tabular}

also fit gender-specific Poisson regression models for excess mortality adjusting for follow-up time, period of diagnosis and age diagnosis ${ }^{(10)}$. This model was estimated in the framework of generalized linear models assuming a poisson distribution for the observed numbers of deaths.

Statistical analysis was done in STATA, SAS 9.2 and Excel.

\section{Results}

Frequency of sinonasal malignancies

Using the SCR a total of 3,221 patients with non-lymphoid malignant tumours of the sinonasal cavity and the paranasal sinuses were identified (Table 1). The majority were diagnosed in the sixth decade or later. SNMM had the highest average age at diagnosis of 71.7 years in contrast to adenoidcystic cancer that had the lowest mean age at diagnosis, 62.5 years.

The predominant histopathological diagnosis was SCC followed by adenocarcinoma and SNMM. A majority of patients with SNM were male. $65 \%$ of the patients with SCC were male, but for SNMM and adenoid cystic carcinoma, the women predominated (55\% and $53 \%$, respectively)(Table 2 ).

In total, $50.5 \%$ of the tumours involved the nasal cavity, and $31.7 \%$ affected the maxillary sinus. The remaining tumours were localized in the other sinuses or had a spread growth. Over the years, we find an increasing proportion tumours originating from the nasal cavity compared to tumours from the maxillary sinuses. There were no differences in localization between genders. However, we found that SNMM had a higher frequency of tumours located in the nasal cavity compared to SCC (Table 2).

Incidence of sinonasal malignancies

For SNM the overall incidence rate per 100,000 decreased from
1.19 in the 1960 's to 0.86 in the 2000 's. The incidence trend for men and women did not differ over the years (Figure 1).

SCC as well as undifferentiated carcinoma decreased whereas the incidence of adenocarcinoma increased in the beginning of the study period and decreased at the end. SNMM is the only sinonasal malignancy that has increased in incidence during the study period (from 0.04 1960-1964 to 0.15 2005-2010) (Figure 2a-e).

\section{Survival of sinonasal malignancies}

We evaluated the 5-year relative survival data for all patients from 1960 to 2010 . In total, 36 patients were lost to follow-up. We found that women had a $4 \%$ lower excess mortality than men, but this was not statistically significant $(p=0.48)$. During the study period, the survival improved over time for both genders regardless the histological diagnosis (Figure 3 and 4).

The patients with adenocarcinoma and SCC had a 5-year survival of $56 \%$ and $46 \%$, respectively. SNMM and undifferentiated carcinoma had a poorer prognosis, $27 \%$ and $37 \%$, respectively. Patients with adenoid cystic cancer had the best 5 -year progno-

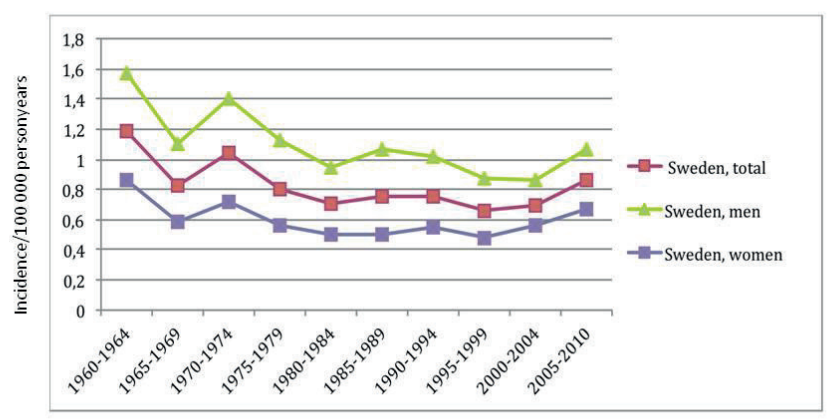

Figure 1. Incidence of sinonasal malignancies in Sweden, 1960 - 2010. 


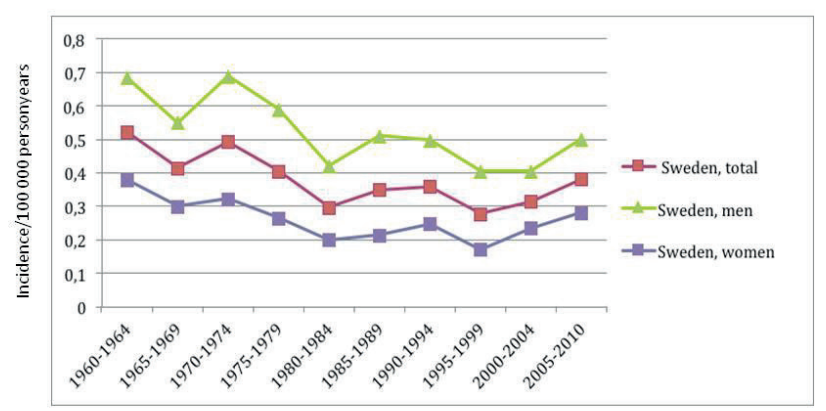

Figure 2a. Incidence of SSC in Sweden, 1960 - 2010.

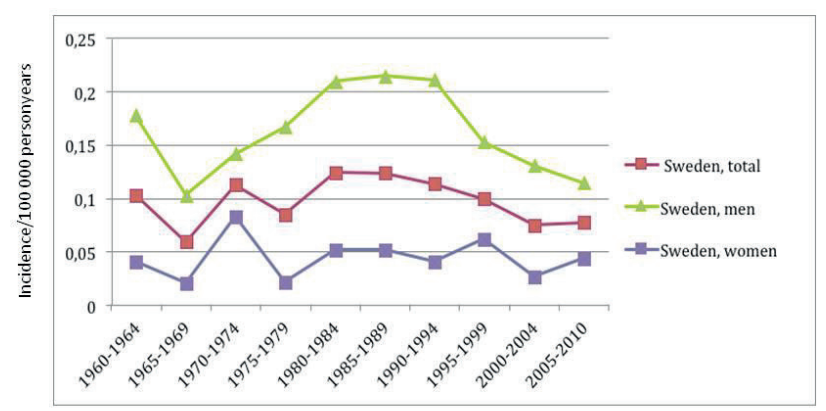

Figure 2b. Incidence of adenocarcinoma in Sweden, 1960 - 2010.

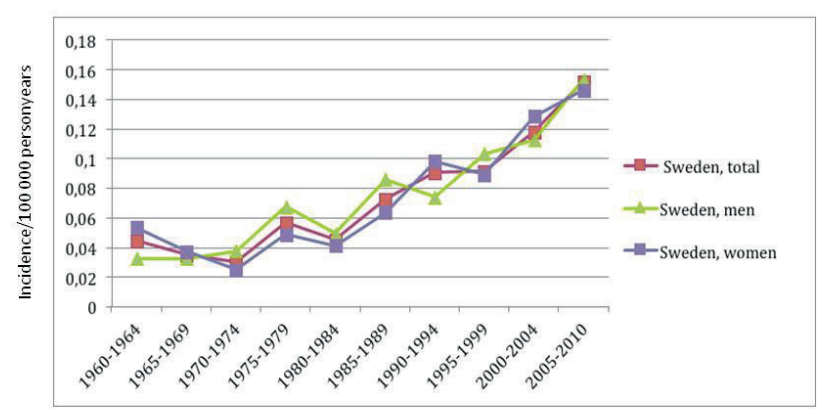

Figure 2c. Incidence of SNMM in Sweden, 1960 - 2010.

sis, $58 \%$.

Patients with tumour originating from the nasal cavity have a better prognosis (mean overall survival 84.2 months) than patients with tumours localized in the maxillary sinuses (mean overall survival 55.2 months) $(p<0.0001)$.

\section{Discussion}

In our study, which is one of the largest population based studies of SNM in recent years, we found that the overall incidence for SNM in Sweden has decreased during the study period 1960 through 2010. However, SNMM's are the only SNM that has increased significantly in the same time period. We found no improvement in the survival of patients with SNM over the last 50 years.

The most frequent sinonasal malignancy during the study period was SCC followed by adenocarcinoma, which also recently has been shown in a large international comparison

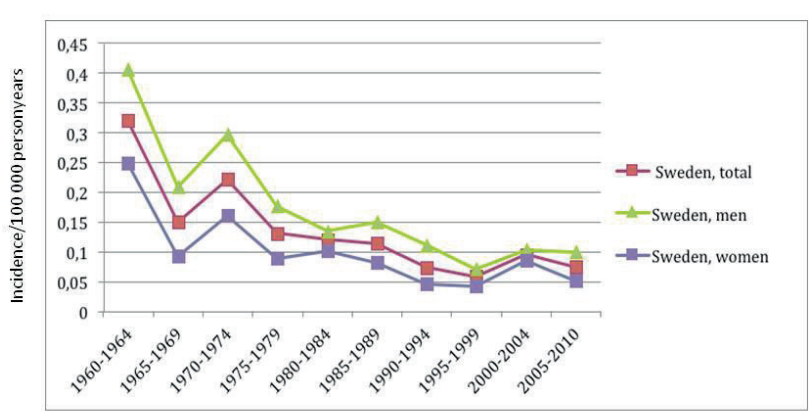

Figure 2d. Incidence of undifferentiated carcinoma in Sweden, 1960 2010.

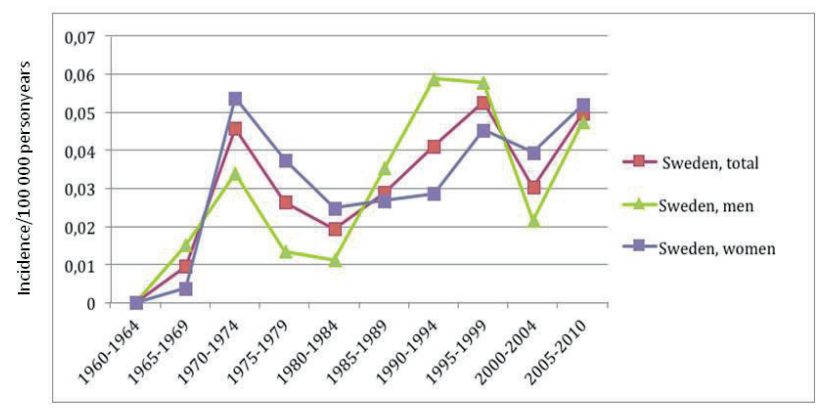

Figure 2e. Incidence of adenoid cystic cancer in Sweden, 1960 - 2010.

of the incidence and mortality of sinonasal cancer ${ }^{(12)}$. However, when comparing incidence data from 1960 and 2010, SCC was still the most frequent tumour followed by SNMM, which was rare in 1960. This increase in the incidence of SNMM has recently been observed in other studies ${ }^{(2,13,14)}$. The differences in distribution over time of the various histological types might reflect an actual increase and decrease of different histological types but also improved diagnostic methods. Presumably, new diagnostic tools such as immunohistochemical markers have increased the accuracy of identifying certain tumours, where undifferentiated tumours in 1960 now are likely to have a specified histological diagnosis. However, for SNMM's the increased incidence may not only be explained by the improvement of diagnostic methods ${ }^{(2)}$. The number of cutaneous malignant melanomas continues to increase in many parts of the world, whereas incidence of mucosal melanomas such as vulvar and ano-rectal melanoma, shows a more complex pattern with a decreasing ${ }^{(1)}$ or stable ${ }^{(2,3)}$ incidence rate.

An association between sinonasal malignancies and exposure to inhaled carcinogens such as wood dust has been described in several studies published in the late 1960 s and early $1970 \mathrm{~s}^{(15,16)}$. Adenocarcinoma of the nasal cavity or sinuses is strongly related to exposure to dust from hard wood or leather dust ${ }^{(17,18)}$. It has been suggested that occupational exposure to formaldehyde might induce SNMM ${ }^{(19)}$. Heavy air pollution has been described to be an etiological factor for SNM in general ${ }^{(18,20)}$. It may be hypothesised that the decreasing incidence in most of the different histological types of SNM may be correlated to better working conditions and reduced air pollution over the years. 


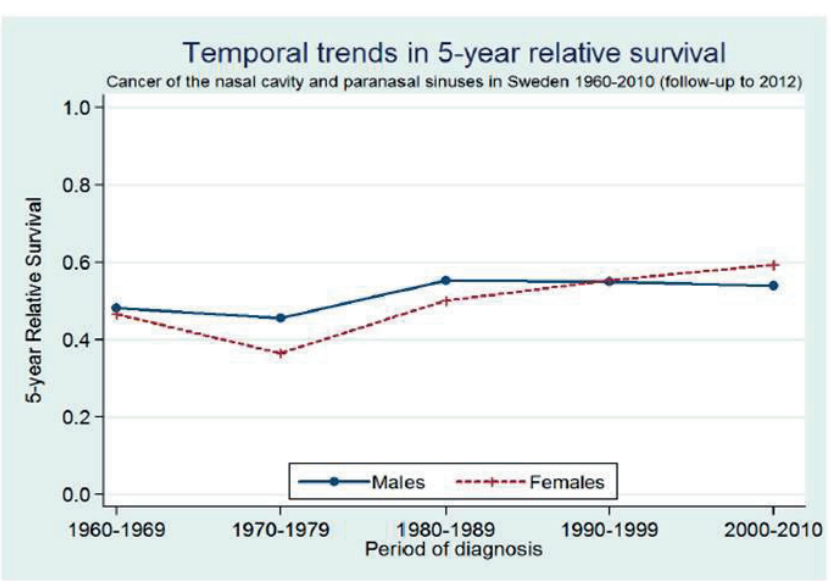

Figure 3. Temporal trends in 5-year relative survival stratified to gender.

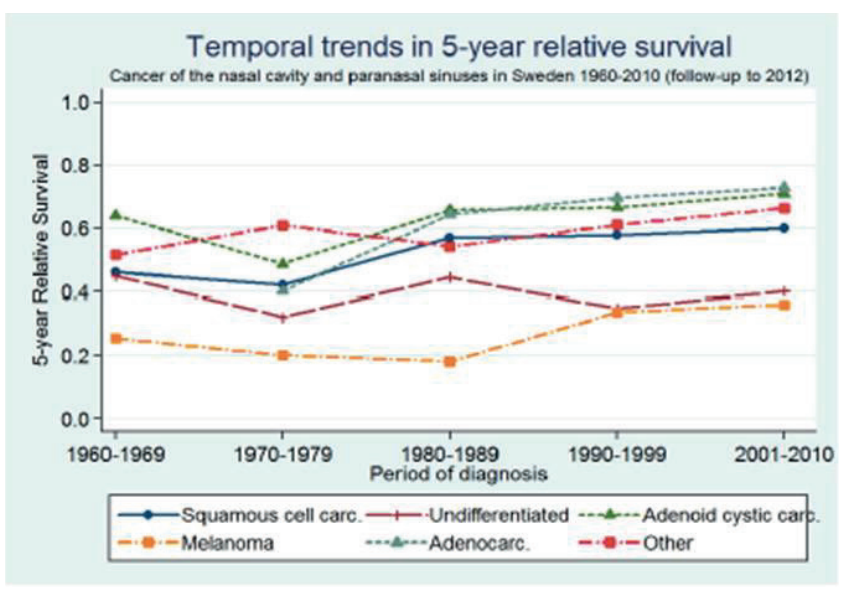

Figure 4. Temporal trends in 5-year relative survival stratified to cancer type.
The decreasing incidence for SCC might be due to reduced occupational exposure and smoking habits that we know play a crucial role in the pathogenesis ${ }^{(4)}$. Interestingly, though, our data does not show any clear-cut difference in 5-year survival between men and women in contrast to other authors ${ }^{(5)}$. This has also been confirmed by others in long-term survival analy$\mathrm{sis}^{(21)}$, where survival is proposed to be linked to stage at diagnosis, extent of spread, onset of symptoms rather than gender. The proportion tumours in the nasal cavity $(50.5 \%)$ were similar to other studies ${ }^{(4,5,12)}$. It would be lower if it were not for the SNMM's, which are more frequent in the nasal cavity. The increasing proportion tumours originating from the nasal cavity compared to the maxillary sinuses for all SNM over the study period was also found in a recent published article by Youlden and colleagues ${ }^{(12)}$. The reason for this is unknown. Possibly patients' and doctors' delay in the 1960's may have resulted in a misclassification of the tumour in the 1960's compared to the 2010's, where the better diagnostic tools and earlier diagnosis gives a more reliable origin of the tumour. Some of the tumours in the nasal cavity (SCC and SNMM) may be misclassified as they might originate from the skin in the vestibulum nasi. It might also have been difficult to differentiate between primary tumours originating from the nasal cavity, the ethmoid sinuses and maxillary sinuses before the modern diagnostic tools became available (CT and MRI).

We also found a significant better survival for patients with tumours originating from the nasal cavity compared to other location, which is consistent with other studies ${ }^{(5,6,22)}$, presumably because tumours from the nasal cavity present earlier with symptoms such as nasal blockage and are therefore earlier diagnosed.

Even though the proportion of SNMM has increased, there is a slight increase in overall relative 5-year survival. Surprisingly, the new treatment strategies and modern diagnostic tools, which facilitate a greater precision of therapy have not significantly ameliorated treatment outcome.

Unfortunately, we were not able to retrieve data on how the tumour stage changed over time, although a recent study by Jangard and colleagues has shown that SNMM tend to have an increased number of more advanced tumours over the years in Sweden ${ }^{(2)}$.

The strength of our study, which is the largest European population based study on SNM of its kind, is that it is based on data from a nationwide population based cancer registry, SCR, with a uniquely high coverage rate ${ }^{(7,8)}$. Furthermore, our study is the first to describe the trends in incidence and survival of sinonasal malignancies in Sweden over a long period. The weakness is that due to the rarity SNM and even more in some of the histological subtypes, conclusions from this material may difficult.

\section{Conclusion}

In conclusion, as a group SNM in Sweden have decreased over the last 50 years. However, SNMM have increased and is now the second most common SNM. Despite improvement in prognosis in other malignancies treatment of SNM still has a poor survival outcome.

\section{Acknowledgement}

Financial support provided by: Grants from the Stockholm County Council (ALF-funding), Karolinska Institutet and the ACTA Otolaryngologica Foundation, Nachmanson Foundation.

\section{Authorship contribution}

AE: Collecting data, study design, statistical analysis, manuscript writing, correspondence; MJ: Study design, statistical analysis, manuscript writing; NH and PD: Statistical analysis, manuscript revision; LM, LHN, PS: Study design, manuscript revision.

\section{Conflicts of interest}

No conflict of interest. 


\section{References}

1. The Swedish National Cancer Registry. Cancer incidence in Sweden, 1960-2010. Stockholm: National Board of Health and Welfare, annual publications 1960-2010.

2. Jangard M, Hansson J, Ragnarsson-Olding B. Primary sinonasal malignant melanoma: a nationwide study of the Swedish population, 1960-2000. Rhinology. 2013; 51: 22-30.

3. Norlander T, Frodin JE, Silfversward C, Anggard A. Decreasing incidence of malignant tumors of the paranasal sinuses in Sweden. An analysis of 141 consecutive cases at Karolinska Hospital from 1960 to 1980. Ann Otol Rhinol Laryngol. 2003; 112: 236-241.

4. Kuijpens JH, Louwman MW, Peters R, Janssens GO, Burdorf AL, Coebergh JW. Trends in sinonasal cancer in The Netherlands: more squamous cell cancer, less adenocarcinoma. A population-based study 1973-2009. Eur J Cancer. 2012; 48: 2369-2374

5. Turner JH, Reh DD. Incidence and survival in patients with sinonasal cancer: a historica analysis of population-based data. Head Neck. 2012; 34: 877-885.

6. Grau C, Jakobsen MH, Harbo G, SvaneKnudsen V, Wedervang K, Larsen SK, et al. Sino-nasal cancer in Denmark 1982-1991--a nationwide survey. Acta Oncol. 2001; 40: 19-23.

7. Barlow L, Westergren $K$, Holmberg L, Talback M. The completeness of the Swedish Cancer Register: a sample survey for year 1998. Acta Oncol. 2009; 48: 27-33.

8. Mattsson B, Wallgren A. Completeness of the Swedish Cancer Register. Non-notified cancer cases recorded on death certificates in 1978. Acta Radiol Oncol. 1984; 23: 305313.

9. Dickman PW, Adami HO. Interpreting trends in cancer patient survival. J Int Med. 2006; 260: 103-117.

10. Dickman PW, Sloggett A, Hills M, Hakulinen T. Regression models for relative survival. Stat Med. 2004; 23: 51-64.
11. Brenner H, Arndt V, Gefeller O, Hakulinen T. An alternative approach to age adjustment of cancer survival rates. Eur J Cancer. 2004; 40: 2317-2322.

12. Youlden DR, Cramb SM, Peters S, Porceddu SV, Moller H, Fritschi L, et al. International comparisons of the incidence and mortality of sinonasal cancer. Cancer Epidemiol. 2013; 37: 770-779.

13. Marcus DM, Marcus RP, Prabhu RS, Owonikoko TK, Lawson DH, Switchenko $J$, et al. Rising incidence of mucosal melanoma of the head and neck in the United States. J Skin Cancer. 2012;2012:231693.

14. Koomen ER, de Vries E, van Kempen LC, van Akkooi AC, Guchelaar HJ, Louwman MW, et al. Epidemiology of extracutaneous melanoma in the Netherlands. Cancer epidemiology, biomarkers \& preventionCancer Epidemiol Biomarkers Prev. 2010; 19: 14531459.

15. Ragnarsson-Olding $B$, Johansson H, Rutqvist LE, Ringborg U. Malignant melanoma of the vulva and vagina. Trends in incidence, age distribution, and long-term survival among 245 consecutive cases in Sweden 19601984. Cancer. 1993; 71: 1893-1897.

16. Weinstock MA. Malignant melanoma of the vulva and vagina in the United States: patterns of incidence and population-based estimates of survival. Am J Obstet Gynecol. 1994; 171: 1225-1230.

17. Ragnarsson-Olding BK, Nilsson PJ, Olding LB, Nilsson BR. Primary ano-rectal malignant melanomas within a population-based national patient series in Sweden during 40 years. Acta oncologica. 2009; 48: 125-131.

18. Acheson ED, Cowdell RH, Hadfield E, Macbeth RG. Nasal cancer in woodworkers in the furniture industry. Br Med J. 1968; 2: 587-596.

19. Acheson ED, Cowdell RH, Rang E. Adenocarcinoma of the nasal cavity and sinuses in England and Wales. Br J Ind Med. 1972; 29: 21-30

20. Hayes RB, Gerin M, Raatgever JW, de Bruyn A. Wood-related occupations, wood dust exposure, and sinonasal cancer. Am J Epid. 1986; 124: 569-577.

21. d'Errico A, Pasian S, Baratti A, Zanelli R, Alfonzo S, Gilardi L, et al. A case-control study on occupational risk factors for sinonasal cancer. Occup Environ Med. 2009; 66: 448-55.

22. Holmstrom M, Lund VJ. Malignant melanomas of the nasal cavity after occupational exposure to formaldehyde. $\mathrm{Br}$ J Ind Med. 1991; 48: 9-11.

23. Calderon-Garciduenas L, Delgado $R$, Calderon-Garciduenas A, Meneses A, Ruiz LM, De La Garza J, et al. Malignant neoplasms of the nasal cavity and paranasal sinuses: a series of 256 patients in Mexico City and Monterrey. Is air pollution the missing link? Otolaryngol Head Neck Surg. 2000; 122: 499-508.

24. Sanghvi S, Khan MN, Patel NR, Yeldandi S, Baredes S, Eloy JA. Epidemiology of sinonasal squamous cell carcinoma: a comprehensive analysis of 4994 patients. Laryngoscope. 2014; 124: 76-83.

25. Harbo G, Grau C, Bundgaard T, Overgaard M, Elbrond O, Sogaard H, et al. Cancer of the nasal cavity and paranasal sinuses. A clinico-pathological study of 277 patients. Acta Oncol. 1997; 36: 45-50.

\author{
Alexandra Elliot, MD \\ Department of Oto-Rhino-Laryngolo- \\ gy, Head and Neck Surgery \\ Karolinska University Hospital \\ Karolinska Institutet \\ 17176 Stockholm \\ Sweden
}

Tel: +46-8-51770000, +46-704334635 